\title{
Global public health leaders responding to the 2009 influenza a HINI virus pandemic
}

Volume 5 Issue I - 2017

Keywords: H1N1 virus, Flu virus, Influenza, Disease control and prevention, Symptoms, Public health

Abbreviations: $\mathrm{CDC}$, Centers for Disease Control and Prevention; WHO, World Health Organization; H, Hemagglutinin; N, Neuraminidase

\section{Editorial}

During March 2009, Mexico experienced a seasonally late peaked incidence of influenza cases in comparison to the usual pattern of manifestation. Moreover, the flu virus which usually harmed very young or old vulnerable patients severely attacked healthy young adults.

The 2009 influenza A H1N1 virus continued to infect the human population throughout Mexico. Since this country is a popular vacation destination favored among tourists traveling from diverse regions across the globe, this infectious agent had the potential to quickly spread throughout the world and hazardously affect millions of lives, for example: Subsequent similar signs and symptoms of influenza outbreaks quickly spread throughout the southwest region of the United States. Test samples from these cases analyzed by virologists from the Centers for Disease Control and Prevention (CDC) revealed that the same strain of the 2009 influenza A H1N1 virus had infected both American and Mexican patients.

While attending the $12^{\text {th }}$ World Congress on Public Health in Istanbul, Turkey during late April 2009, I personally witnessed public health leaders urgently having to leave to implement global health safety precautions in order to prevent a worldwide pandemonium and the impending doom of an uncontrollable 2009 influenza A H1N1 virus pandemic. Their work helped to prevent the reoccurrence of the 1918 influenza A H1N1 virus pandemic which eradicated more lives in comparison to the number of soldiers who died during World War I resulting in a global morbidity and mortality rate of 500 million and 50 to 100 million individuals, respectively.

Dr. Margaret Chen, Director General of the World Health Organization (WHO) had to cancel her guest appearance at the 12th World Congress on Public Health as a keynote speaker in order to save millions of lives through global pandemic preparedness and response, for example: Airport employees working across the globe were mandated to wear masks and gloves to help prevent a worldwide transmission of the flu virus through passengers traveling to various destinations. Understanding that the conference attendees would be disappointed by her absence, Dr. Chen spoke via video about the importance of her immediate attention to global health preventive initiatives especially since the exact flu viral strain and its' mode of transmission and genome replication were unconfirmed. She understood that many individuals of the general population who were non-health care/public health professionals believed that it was a nuisance for them of having to adhere to the preventive safety precautions; they did not understand the benefits of the global preventive health initiatives being rendered to them. Even though she realized that murmuring would occur among some individuals of the general population, Dr. Chen adhered to her principles of governing global preventive health practices while demonstrating the characteristics of a brave and fearless leader of public health; she put the well-being of the global community at the forefront instead of succumbing to the unwarranted suspicions and complaints from individuals not adequately well informed in her perspective field of study. I witnessed firsthand the courage of a world public health leader serve the wellbeing of the global community with great efficiency during an urgent state of affairs.

The genome of the 2009 influenza A H1N1 virus differed in composition in comparison to the swine flu virus responsible for the 1918 and 1976 pandemics. According to scientists from the Center for Disease Control and Prevention (CDC) and the Icahn School of Medicine at Mount Sinai Center for Research on Influenza Pathogenesis, the genome of the 2009 influenza A H1N1 virus was the reassortment of a swine flu virus circulating in the Americas especially North America jumping between pigs, birds, and humans and an Eurasian swine flu virus circulating many years through pigs in Mexico before jumping into humans.

Scientists knew that the surface proteins hemagglutinin $(\mathrm{H})$ and neuraminidase $(\mathrm{N})$ of the influenza A H1N1 virus enabled this infectious agent to adhere to and invade cells of the respiratory tract, respectively. These surface proteins which were produced under hereditary influence had undergone genetic reassortment involving antigenic drifts and shifts led to the production of a new viral strain infecting the human population who lacked immunity. The development and administration of the 2009 flu pandemic vaccine provided the much needed immunity for the human population to be protected against the new viral strain of the 2009 influenza A H1N1 virus.

It was an interesting experience to observe health care/public health specialists, scientists, and leaders collaboratively engage in global pandemic preparedness and response to protect the wellbeing 
of the global community which included the developed as well as developing countries around the world.

\section{Acknowledgments}

None.

\section{Conflicts of interest}

None. 